\title{
Biomacromolecular Stereostructure Mediates Mode Hybridization in Chiral Plasmonic Nanostructures
}

\author{
Calum Jack, ${ }^{\dagger}$ Affar S. Karimullah, ${ }^{*},{ }^{\dagger}$, Ross Leyman, $^{\dagger}$ Ryan Tullius, $^{\dagger}$ Vincent M. Rotello, $^{\S}$ Graeme Cooke, $^{\dagger}$ \\ Nikolaj Gadegaard, ${ }^{\ddagger}$ Laurence D. Barron, ${ }^{\dagger}$ and Malcolm Kadodwala ${ }^{, \dagger}$ \\ ${ }^{\dagger}$ School of Chemistry, Joseph Black Building, University of Glasgow, Glasgow G12 8QQ United Kingdom \\ ${ }^{*}$ School of Engineering, Rankine Building, University of Glasgow, Glasgow G12 8LT, United Kingdom \\ ${ }^{\S}$ Department of Chemistry, 710 N. Pleasant Street, University of Massachusetts, Amherst, Massachusetts 01003, United States
}

\section{Supporting Information}

\begin{abstract}
The refractive index sensitivity of plasmonic fields has been exploited for over 20 years in analytical technologies. While this sensitivity can be used to achieve attomole detection levels, they are in essence binary measurements that sense the presence/absence of a predetermined analyte. Using plasmonic fields, not to sense effective refractive indices but to provide more "granular" information about the structural characteristics of a medium, provides a more information rich output, which affords opportunities to create new powerful and flexible sensing technologies not limited by the need to synthesize chemical recognition elements. Here we report a new plasmonic phenomenon that is sensitive to the biomacromolecular structure without relying on measuring effective refractive indices.

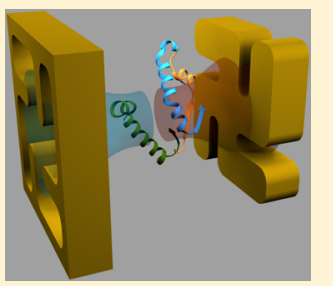
Chiral biomaterials mediate the hybridization of electric and magnetic modes of a chiral solid-inverse plasmonic structure, resulting in a measurable change in both reflectivity and chiroptical properties. The phenomenon originates from the electricdipole-magnetic-dipole response of the biomaterial and is hence sensitive to biomacromolecular secondary structure providing unique fingerprints of $\alpha$-helical, $\beta$-sheet, and disordered motifs. The phenomenon can be observed for subchiral plasmonic fields (i.e., fields with a lower chiral asymmetry than circularly polarized light) hence lifting constraints to engineer structures that produce fields with enhanced chirality, thus providing greater flexibility in nanostructure design. To demonstrate the efficacy of the phenomenon, we have detected and characterized picogram quantities of simple model helical biopolymers and more complex real proteins.
\end{abstract}

KEYWORDS: Plasmonic hybridization, biosensing, chiral plasmonics, optically active second harmonic generation

$\mathrm{T}_{\mathrm{i}}$ he ultrasensitive ( $\leq$ picogram) detection and characterization of biomacromolecules is a critical capability with applications in medical diagnostics, pharmaceutical development, and biochemical research. The sensitivity of the spectral position of a plasmonic resonance to the effective refractive index within the near-field volume has been exploited for ultrasensitive detection. ${ }^{1,2}$ However, these evanescent plasmonic fields are not intrinsically sensitive to macromolecular structure. Consequently, plasmonic fields act as a transduction element, while identification of a target biomolecule is achieved by functionalizing a plasmonic structure with an appropriate receptor molecule. ${ }^{2,3}$ Development of appropriate receptors for plasmonic sensors is a time-consuming and costly endeavor, ultimately being the limiting step in defining applications. In contrast chiroptical spectroscopy, based on the differential interaction of circularly polarized light $(\mathrm{CPL})$ is a rapid and generic tool for characterizing and identifying biological materials. ${ }^{4}$ Unfortunately, chiroptical spectroscopic methods have sensitivities $>6$ orders of magnitude less than plasmonic refractive index sensors. Effectively combining the (structural) fingerprinting capabilities of chiroptical spectroscopy with the detection levels of plasmonic fields would create a flexible and generic sensing paradigm, which would not be limited by developing appropriate receptor chemistry. Recent reports on off-resonance excitations of chiral molecules coupled to plasmonic resonators and evanescent fields with enhanced chiral asymmetry (referred to as superchiral fields) generated around chiral plasmonic structures have been used for enhanced sensing of chiral molecules. ${ }^{5-10}$ In particular, the latter has been used in a form of polarimetry (superchiral polarimetry) that provides a level of structural characterization at the subpicogram level. However, the level of the structural fingerprinting is limited; crucially superchiral polarimetry measures the chiral structural anisotropy rather than secondary structure content directly. Consequently, superchiral polarimetry is a less incisive probe of biomacromolecular structure than conventional chiroptical spectroscopy. This is illustrated by the fact that two proteins with similar secondary structure can give different superchiral polarimetry response. ${ }^{10,11}$ The difference in the structural incisiveness of conventional chiroptical spectroscopy and superchiral polarimetry derives from the origins of the optical active (chiral dependent) responses. In the case of superchiral polarimetry, optical activity is primarily derived from the quadrupolar response $\left(E_{2}\right)$ of a

Received: June 21, 2016

Revised: August 19, 2016

Published: August 22, 2016 
(A)

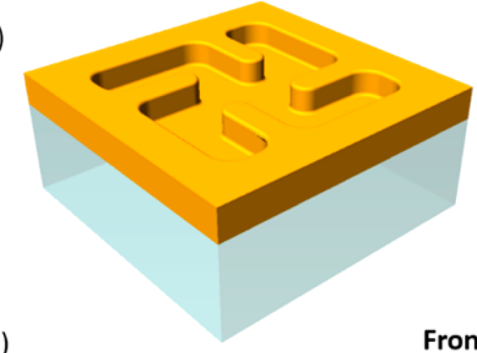

(B)

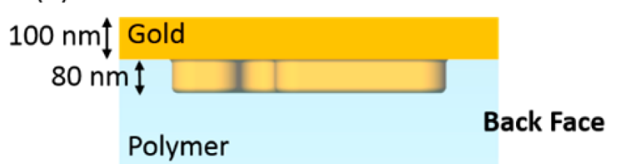

(C)

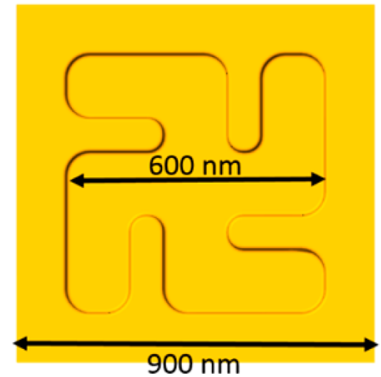

(D)

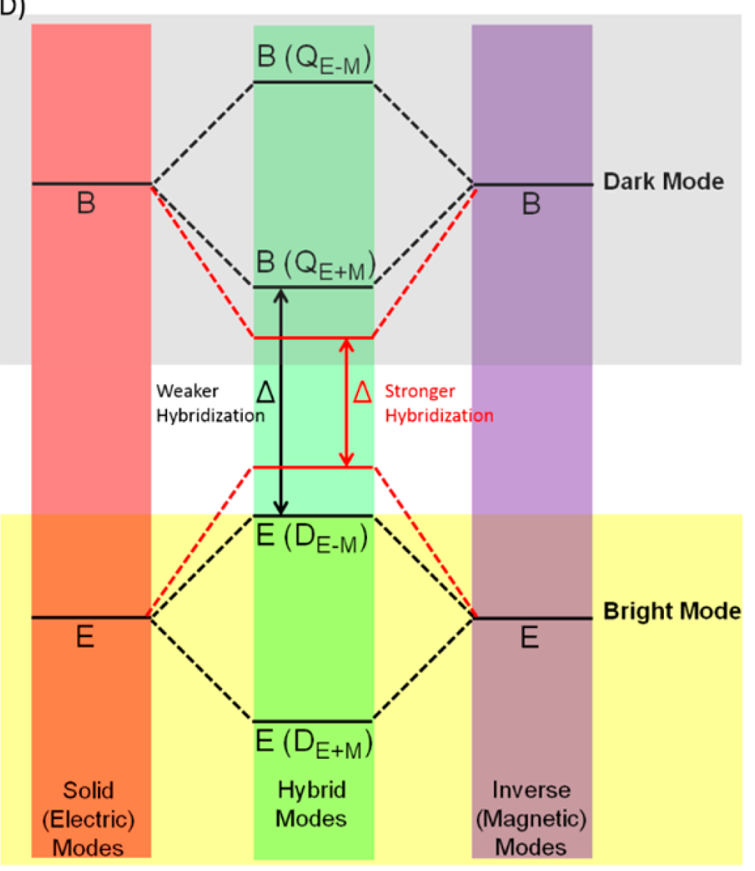

Figure 1. Geometrical description of a nanostructure on the TPS. (A) Perspective view, (B) side view, and (C) top view. (D) The molecular orbital schematic for the nanostructure derived from the symmetry properties. The red lines represent the position of the hybrid modes in the presence of adsorbed biomolecules. The red lines for the $\mathrm{E}\left(\mathrm{D}_{\mathrm{e}+\mathrm{M}}\right)$ and $\mathrm{B}\left(\mathrm{Q}_{\mathrm{E}-\mathrm{M}}\right)$ have been removed for clarity.

chiral material, which is only nonzero for anisotropic chiral media. ${ }^{12}$ In contrast the optical activity of conventional chiroptical spectroscopy in isotropic media derives from the electric-dipole $\left(E_{1}\right)$-magnetic-dipole $\left(M_{1}\right)$ cross term, which provides a distinct fingerprint of a secondary and to some extent tertiary structure. ${ }^{4,12}$

In this study, we present a new phenomenon, the mediation of the hybridization of electric and magnetic modes of a hybrid solid-inverse metamaterial, via the interaction of chiral evanescent fields with biomaterials. This new mediation process is significantly different to previous studies that relied on superchiral evanescent fields interacting with chiral structural anisotropy. ${ }^{10}$ It occurs when the sense of the chiral asymmetry of the evanescent fields around the nanostructure matches that of the chiral material. The mediation of the hybridization of the electric and magnetic modes are dependent upon the $E_{1} M_{1}$ response of the chiral material, and thus the effect provides a distinct fingerprint of secondary structure motifs in an isotropic system. The effect can occur when fields are weakly chiral, that is, the evanescent fields have a smaller chiral asymmetry than CPL; we refer to these fields as subchiral.

Central to our observation is the use of plasmonic solidinverse hybrid gold metafilms that allow the hybridization of symmetrically equivalent electric and magnetic modes. The metafilms are formed on a nanostructured templated polycarbonate substrate (TPS). ${ }^{13}$ The TPS is indented with a square array with a periodicity of $900 \mathrm{~nm}$ of left/right handed $(\mathrm{LH} / \mathrm{RH})$ gammadions (lateral dimensions $600 \mathrm{~nm}$ ) and are coated with $100 \mathrm{~nm}$ Au by evaporation, Figure 1. The TPSs are hybrid solid-inverse structures consisting of a solid nanostructure and an identical shaped void (inverse structure) directly above it. In line with Babinet's principle, the roles of electric and magnetic fields are switched between solid and inverse structures (i.e., the magnetic, rather than an electric, dipole transition is the lowest energy excitation in a void). ${ }^{14}$ The implications of this are that symmetry equivalent electric and magnetic modes of the solid and inverse structures are spatially located directly above each other and can consequently couple in an analogous manner to hybridization of orbitals in molecular systems (i.e., electric and magnetic modes can combine either in or out-of-phase). Analogous to molecular systems, ${ }^{15-18}$ an "orbital" hybridization diagram can be constructed through the application of symmetry analysis, Figure 1 (and Supporting Information 1.1). The gammadion structure has hybridized modes which are dipolar $\left(D_{\mathrm{E}-\mathrm{M}}\right.$ and $\mathrm{D}_{\mathrm{E}+\mathrm{M}}$ ) with $\mathrm{E}$ symmetry, optically bright, and quadrupolar $\left(\mathrm{Q}_{\mathrm{E}-\mathrm{M}}\right.$ and $\left.\mathrm{Q}_{\mathrm{E}+\mathrm{M}}\right)$ with B symmetry, optically dark, which cannot be directly excited by light (see Supporting Information 1.1).

It is well established that the presence of both bright and dark modes strongly influence the reflectance/transmission properties of metamaterials due to the occurrence of interference phenomena such as Fano resonances and electron induced transparency (EIT)..$^{14,19-21}$ Fano-like resonances, which have characteristic narrow asymmetric line shapes, occur when there is relatively weak interference between the bright and dark modes. ${ }^{22}$ EIT, which manifests as an increase in reflectance within a reflectivity dip, occurs when there is stronger coupling between dark and bright modes. For the current solid-inverse structures, the level of coupling is governed by the energy separation, $\Delta$, Figure 1D, of the bright and dark, $\mathrm{E}_{\mathrm{E}-\mathrm{M} \text { and }} \mathrm{B}_{\mathrm{E}+\mathrm{M}}$, modes. The $\Delta$ separation is dependent upon the level of hybridization of the electric and magnetic modes, greater levels of hybridization lead to smaller $\Delta$ values, as highlighted in red in Figure 1D. The level of hybridization of electric and magnetic modes also directly controls the nature of the chirality displayed by a solid-inverse structure. Low levels of hybridization result in materials that display chirality described 

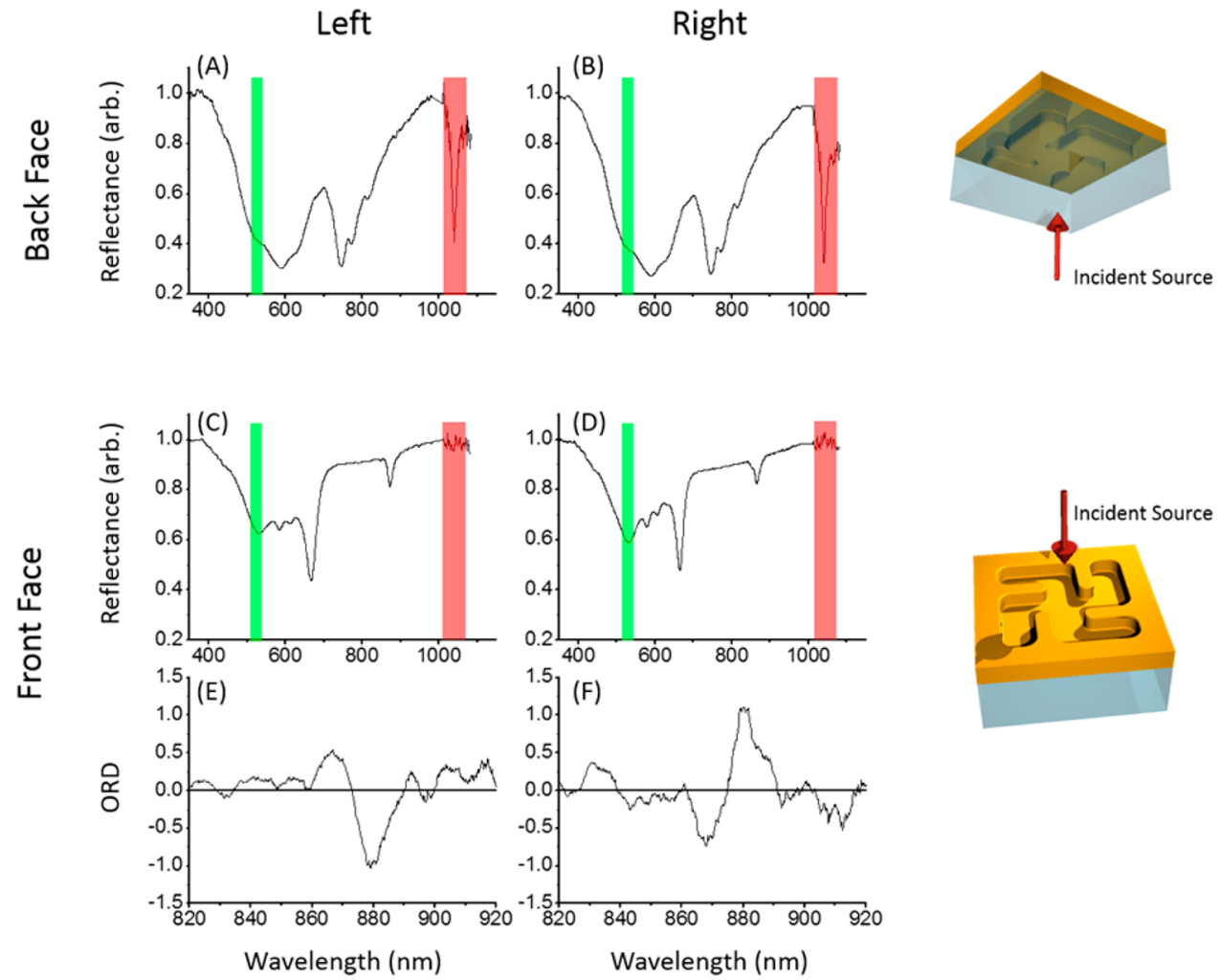

Figure 2. Reflectivity plots for $(A, B)$ back face illuminations and $(C, D)$ front face illumination and $(E, F)$ show the ORD for front face illumination. The red regions show the spectrum covered by the laser source and the green regions show the SHG spectrum.

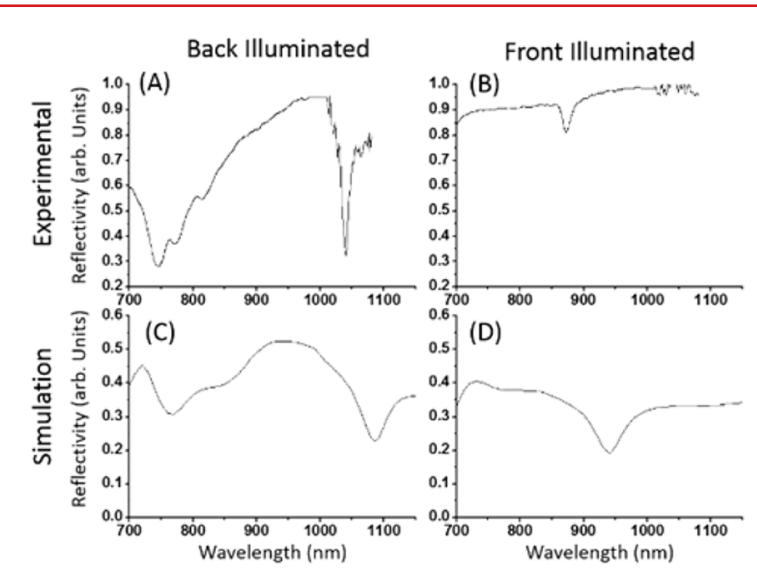

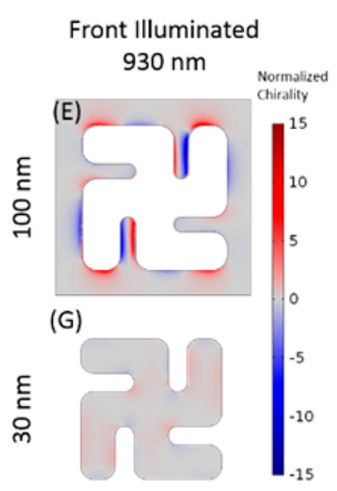

Linear Experiment

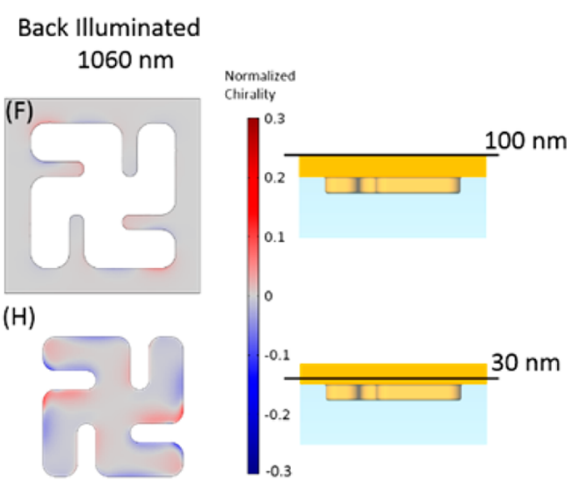

SHG Experiment

Figure 3. (A-B) Experimental reflectivity compared to (C-D) simulated reflectivity. (E) and (G) Normalized chirality surface plots for the front face regions where a surface bound molecule will interact showing large chiral fields when the illumination is from the front face. $(\mathrm{F})$ and $(\mathrm{H})$ display the same region with subchiral fields when back face is illuminated.

by the coupled oscillator model and exhibit weak levels of optical activity (e.g., optical rotation). Strong hybridization in a chiral solid-inverse structure leads to materials that are best described by the helical oscillator model of chirality and display strong levels of optical activity. Consequently, the level of hybridization of modes within a hybrid structure has a direct influence on both the observed reflectivity/transmittance properties and chiroptical response. ${ }^{13}$

In the current study, changes in Fano line shape and chiral properties that would be indicative of a change in hybridization are monitored, respectively, by (linear) reflectivity and nonlinear optically active second harmonic generation (OASHG). Measurements were performed using a range of chiral biopolymers (proteins and polypeptides), and the dependence of the observed phenomenon on macromolecular stereostructure was determined.

We performed reflectivity and SHG experiments on the TPS. In the case of reflectivity, the samples were characterized with light normally incident on the front face and the back face. However, only the front face was exposed to buffer and protein solutions. The reflectivity of front and back faces with buffer is shown in Figure 2A-D. Gammadion enantiomers produce similar near-infrared (NIR) reflectance spectra which possess sharp asymmetric reflectance dips, $\sim 870$ and $1060 \mathrm{~nm}$, typical of Fano-like resonances, consistent with a weak level of hybridization of the electric and magnetic modes. The ORD spectra, Figure 2E,F, are also consistent with weak hybridization in the case when excited from the front face displaying both low 
levels of optical rotation $\left(\sim 1^{\circ}\right)$ and a signature line shape of the coupled oscillator model of optical activity. When light is incident from the back face, no ORD is observed with the sensitivity of our measurements. The poor signal-to-noise of ORD spectra means that they can provide little insight into small changes in the chiroptical properties caused by subtle changes in the hybridization of the substrates induced by the adsorbed biopolymers.

The reflectivity spectrum is reasonably reproduced using EM modeling, Figure $3 \mathrm{~A}-\mathrm{D}$, albeit with a slight red shift. The near fields display chiral asymmetries that have been parametrized using the optical chirality parameter $\mathrm{C}$, first proposed by Tang et al. ${ }^{23,24}$ The chirality of the evanescent fields at the front facing surfaces of TPSs are shown in Figure 3E-H. These are the surfaces where the TPS would be exposed to buffer solutions and surface adsorbed proteins. Scattering of linearly polarized light from a structure generates fields with $\mathrm{LH}$ and $\mathrm{RH}$ chiral asymmetries, albeit with one handedness of field produced in excess; thus the evanescent fields have a net overall chirality. When front illuminated, the resonance at $\sim 870 \mathrm{~nm}$ shows optical activity (Figure 2E,F). The simulations at the corresponding resonance show that the fields here have chiral asymmetries greater than circularly polarized radiation $(C>1)$, that is, they are superchiral. When back illuminated, as would be the case for OA-SHG, the resonance lies at $1060 \mathrm{~nm}$ and shows values of $C<1$, that is, subchiral fields, on the same surfaces.

Reflectivity spectra from LH and RH TPS, at the resonance around $\sim 870 \mathrm{~nm}$, measured from the front face, immersed in solutions of proteins with differing secondary structure motifs, $\alpha$-helical (native bovine serum albumin (BSA)), $\beta$-sheet (Concanavalin A), and disordered structures (denatured-BSA) are shown in Figure 4. None of the proteins have optical absorption peaks in the region of this plasmonic resonance, consequently any change in the Fano resonance is not due to the overlap of a molecular resonance. As expected, due to an increase in local refractive index the absorption of protein causes a red shift of the reflectivity dips for both $\mathrm{LH}$ and $\mathrm{RH}$ substrates $\left(\Delta \lambda_{\mathrm{L} / \mathrm{R}}\right)$. In superchiral polarimetry, the asymmetry in this shift, $\Delta \Delta \lambda=\Delta \lambda_{\mathrm{R}}-\Delta \lambda_{\mathrm{L}}$, is the parameter that is sensitive to chiral structure. ${ }^{10,11}$ For all the proteins studied, a $\Delta \Delta \lambda \approx 0$ is observed. This absence of asymmetry is consistent with the relatively weak net chiral asymmetry of the fields surrounding the gammadion structures, which results in small ORD values by the structures and is reproduced in simulations, Figure 3E,G. Generation of evanescent fields with large chiral asymmetries require efficient coupling of electric and magnetic fields. ${ }^{13,25}$ Solid-inverse structures that display weak hybridization generate chiral fields of lower net chiral asymmetry than from the equivalent structure that are strongly hybridized; they have previously been shown to be ineffective for superchiral polarimetry measurements. ${ }^{13}$

In contrast to $\Delta \lambda_{\mathrm{L} / \mathrm{R}}$ values, protein adsorption does have an asymmetric effect on the line shape of the reflectivity resonances of LH (Figure 4) and $\mathrm{RH}$ substrates (Figure 5). Adsorption of the three protein types has no effect on the line shape of the resonance for the LH substrates but native-BSA and Con $A$ do cause changes for the $\mathrm{RH}$ substrate. In both cases protein adsorption leads to the appearance of a redshifted shoulder on the Fano resonance at $\sim 895 \mathrm{~nm}$ causing an overall broadening of the resonance line shape. In stark contrast, the reflectance line shape remains effectively unchanged upon adsorption of denatured-BSA. The protein-

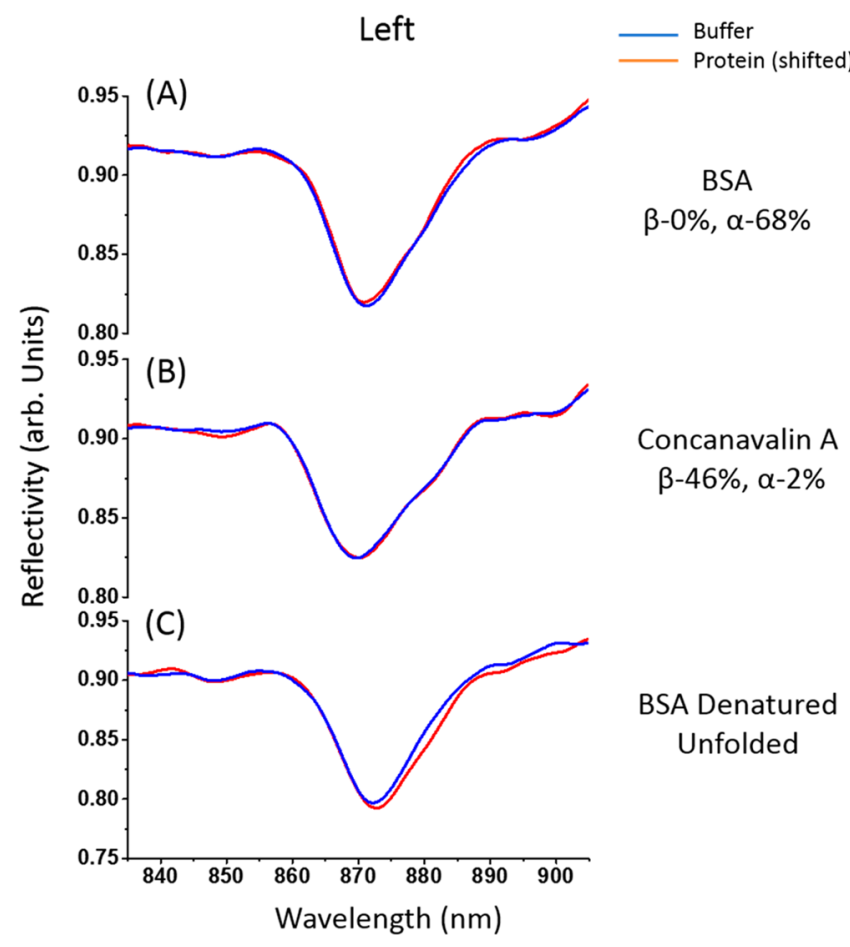

Figure 4. Reflectivity for front illuminated protein experiments on $\mathrm{LH}$ structures. Blue lines are for buffer, and the solid red line is the protein solution data blue-shifted to overlap with the buffer. (A) BSA (B) Concanavalin A, and (C) denatured BSA.

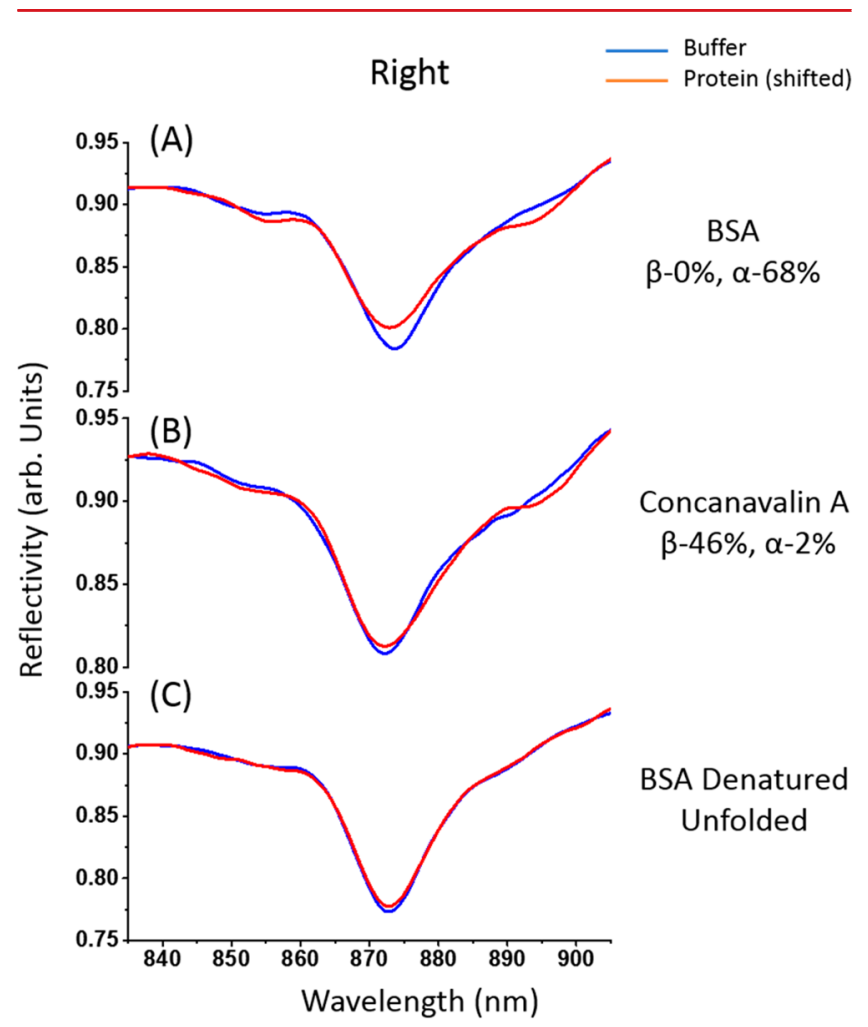

Figure 5. Reflectivity for front illuminated protein experiments on $\mathrm{RH}$ structures. Blue lines are for buffer, and the solid red line is the protein solution data blue-shifted to overlap with the buffer. (A) BSA, (B) Concanavalin A, and (C) denatured BSA. BSA and Concanavalin A show the appearance of the shoulder. 

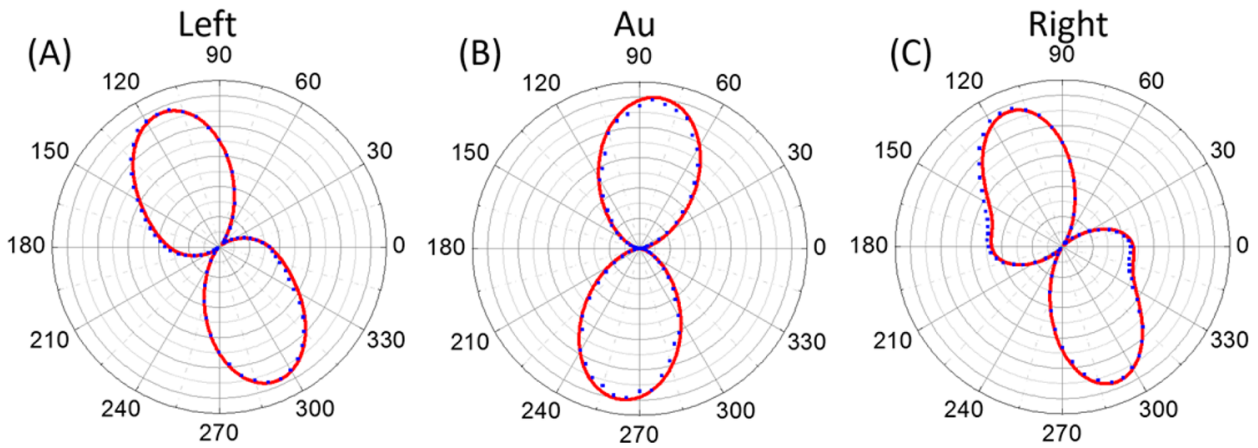

Figure 6. Polar plot of SHG experimental results for S-polarized light incident from the back face for (A) left handed, (B) background Au, and (C) right handed structures with buffer solution on the front end.

induced appearance of a shoulder in the Fano resonance for the RH substrate is consistent with an increase in the coupling (interference) between dark and bright modes, albeit to a lesser degree than is required to induce EIT. In previous studies, a similar shoulder develops on an asymmetric Fano-like peak when the level of coupling between dark (quadrupolar) and bright (dipolar) modes is increased, systematically, by changing the nanostructure geometry. Ultimately, in those previous works the shoulder develops into a reflectivity dip when the nanostructure geometry enables optimal interference between bright and dark modes. ${ }^{13,14,19}$

The reflectivity data we report provides clear evidence that adsorption of $\alpha$-helical and $\beta$-sheet proteins enhances the coupling between the $\mathrm{D}_{\mathrm{E}+\mathrm{M}}$ and $\mathrm{Q}_{\mathrm{E}-\mathrm{M}}$ modes. This indicates a reduction in the $\Delta$ separation value and thus an enhanced level of hybridization of the chiral electric and magnetic modes, implicitly changing the chiral properties of the TPSs.

In a separate series of experiments, the influence of biomolecule adsorption on the front face upon the (nonlinear) chiroptical properties of the back face were probed using OASHG. These measurements have key fundamental differences to the reflectivity and ORD measurements from the front face experiments. The most obvious difference is that the biomolecules have no direct contact with the back face, indeed they are separated from the interface by $100 \mathrm{~nm}$ of Au. Thus, biomolecules can only indirectly influence the chiral properties of the back face, through a mechanism such as the proposed change in hybridization. The second, and a very significant difference, is that the evanescent fields generated at the front face by laser irradiation of the back face $(1060 \mathrm{~nm})$ are only weakly chiral (sub chiral), with $C<1$, Figure $3 \mathrm{~F}-\mathrm{H}$.

Key concepts of OA-SHG from plasmonic nanostructures and the approach to data analysis will be briefly discussed; more detailed discussion can be found elsewhere. ${ }^{26-30}$ Within the dipole approximation, generation of SHG can only arise from noncentrosymmetric environments, consequently for metals such as Au signals can only originate from the surface. However, in the case of a plasmonic nanostructure in which high-intensity fields with steep gradients exist, nondipole contributions can also generate a $\mathrm{SH}$ from the bulk metal. Consequently, the $\mathrm{SH}$ response from plasmonic metamaterials will have a contribution from both the surface and the bulk. In the case of a chiral plasmonic metamaterial, only the surface contribution will display optical activity because the bulk $\mathrm{Au}$ is an achiral structure. OA-SHG is orders of magnitude more sensitive to (interfacial) chirality than linear chiroptical techniques such as ORD.
The form of OA-SHG applied in the current study has been used previously on plasmonic nanomaterials. It involves monitoring a far field $\mathrm{SH}$ signal (S- or P- polarized) as a function of incident linearly polarized radiation. ${ }^{30,31}$ From the symmetry properties of the resulting far field radiation profile, the contributions that $E_{1}, M_{1}, E_{2}$, and higher order excitations make to the $\mathrm{SH}$ intensity can be evaluated. The radiation profiles are fitted with the following function

$$
\begin{aligned}
I_{i}(2 \omega)= & \alpha_{i} \cos ^{4} \theta+\beta_{i} \sin ^{2} \theta \cos ^{2} \theta+\gamma_{i} \sin ^{4} \theta+\delta_{i} \sin \theta \cos ^{3} \theta \\
& +\varepsilon_{i} \sin ^{3} \theta \cos \theta
\end{aligned}
$$

where $i$ is either S- or P-polarization, $\theta$ is the angle between the incident and detected polarizations, and $\alpha_{i}, \beta_{i}, \gamma_{i}, \delta_{i}$, and $\varepsilon_{i}$ are variable fitting parameters. The $\alpha_{i}, \beta_{i}$, and $\gamma_{i}$ values parametrize the $E_{1}, E_{2}$, and $M_{1}$ contribution to the $\mathrm{SH}$ intensity, respectively. The $\delta_{i}$ and $\varepsilon_{i}$ values parametrize the contribution of higher order excitations to the $\mathrm{SH}$ intensity. This formalism was developed from earlier work of Dadap et al. ${ }^{32}$ by Brevet and co-workers for application to solution phase nanoparticles. ${ }^{33}$ It was applied subsequently to planar metamaterials. ${ }^{30,31}$ For molecular chiral systems the $\mathrm{E}_{1}$ response is the dominant contribution to observed optical activity in SHG. However, for plasmonic nanostructures that have intense fields with significant gradients in addition to the dipolar term, quadrupolar (and other higher order) terms can contribute to an asymmetry in the SHG response. Though, only changes in the dipolar contribution $\left(\mathrm{E}_{1}\right)$ are due to surface chirality. ${ }^{29}$

The experimental geometry for the SHG measurement is shown in Supporting Information 1.2. The back-face has a Fano-like resonance dip in reflectivity, $\sim 1040 \mathrm{~nm}$, which is located within the bandwidth $(\sim 80 \mathrm{~nm})$ of the fundamental wavelength of the laser $(1060 \mathrm{~nm})$, Figure 2 . The wavelength of resonance dip at $\sim 1040 \mathrm{~nm}$ is very insensitive to the dielectric environment experienced by the front face, where the proteins are adsorbed. It displays a sensitivity of $3.37 \mathrm{~nm} / \mathrm{RIU}$ (RIU = refractive index units) to the dielectric environment of the front face, which is 2 orders of magnitude smaller ( $373 \mathrm{~nm} / \mathrm{RIU}$ ) than the sensitivity of front face reflectivity dip (Supporting Information 1.3). The EM modeling is in agreement showing no change in field intensities or the chiral evanescent fields for back face illumination when the front face refractive index is varied (Supporting Information 1.3). Consequently, the presence of the biomolecules causes negligible shift in the back face resonance dip. Thus, any change in SHG response after biomolecular adsorption could not originate from a shift in the reflectance dip. 
Table 1. Fitting Parameters for the SHG Experimental Results with Buffer

\begin{tabular}{lccrrr} 
& \multicolumn{4}{c}{ S-polarized, back face, fitting parameters } \\
\hline & $\alpha$ & $\beta$ & $\gamma$ & $\delta$ \\
right & $0.45 \pm 0.02$ & $0.40 \pm 0.07$ & $0.70 \pm 0.02$ & $-0.11 \pm 0.06$ & $-1.17 \pm 0.08$ \\
left & $0.34 \pm 0.02$ & $0.63 \pm 0.07$ & $0.70 \pm 0.02$ & $-0.41 \pm 0.06$ & $0.05 \pm 0.06$ \\
$\mathrm{Au}$ & $0.01 \pm 0.02$ & $0.59 \pm 0.07$ & $0.98 \pm 0.02$ & $0.36 \pm 0.08$ & \\
\hline
\end{tabular}

\section{Left $\quad$ Right}

High $\boldsymbol{\alpha}$-helical
BSA
$\beta-0 \%, \alpha-68 \%$

(A)
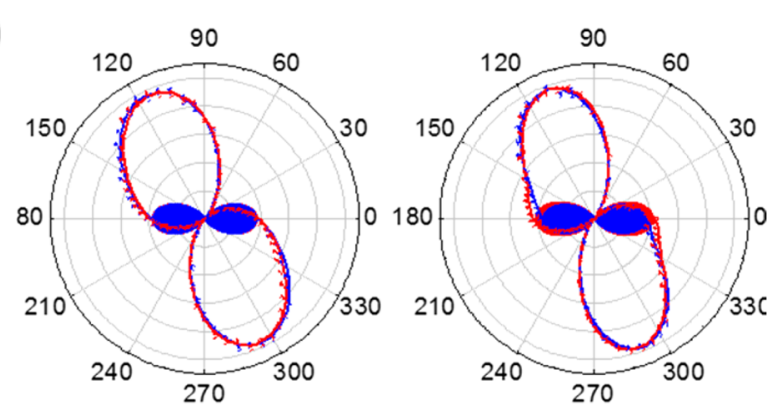

(B)
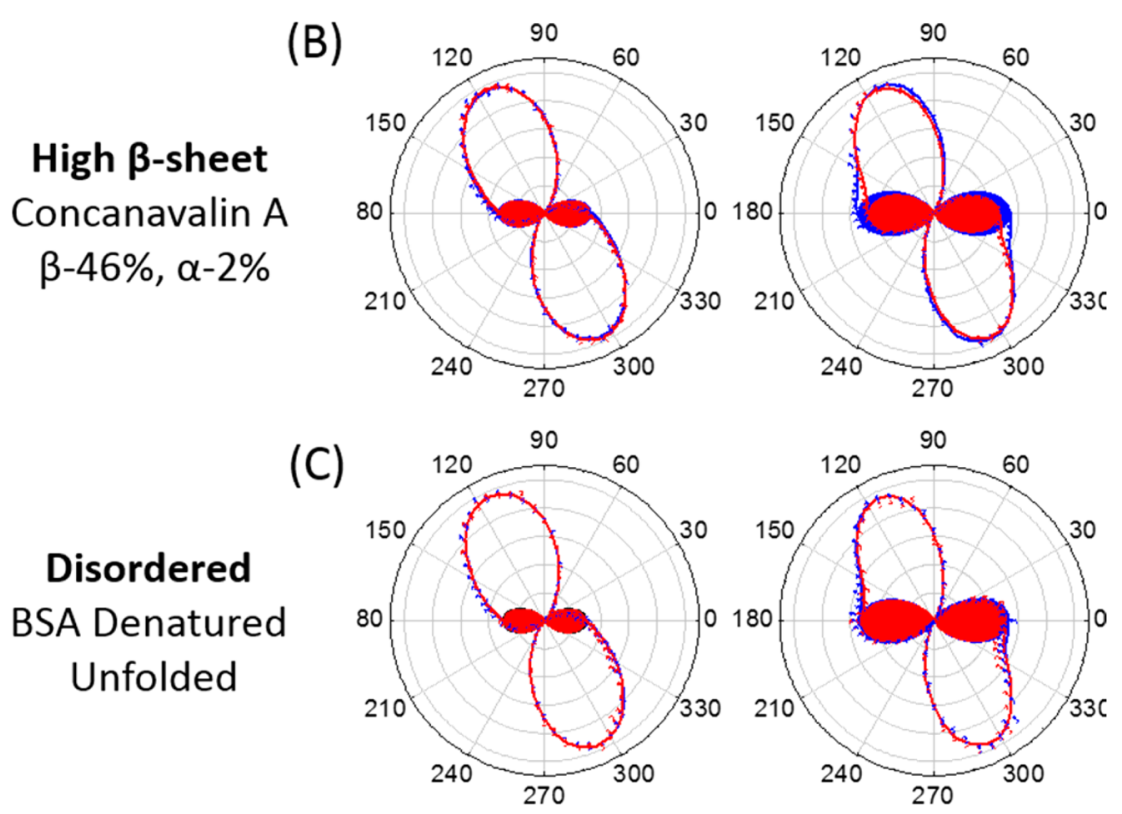

Figure 7. Polar plots showing SHG results for three structurally varying protein solutions for both left handed and right handed structures. Buffer results are in blue and protein results are in red. Experimental results are plotted as points (square markers) while the solid lines show the fitted results. The inner solid parts represent the dipolar contribution ( $\alpha$-value) showing an increase, decrease, or no change due to the protein (solid red) in comparison to the buffer (solid blue). When the $\varsigma$ value is nearly 0 , the protein (red color) is shown on the front. In all other cases, the smaller of the two is shown in the front for clarity.

The resonant nature of the excitation process enhances the sensitivity of the back face SHG to the chirality of the back surface. The far-field $\mathrm{SH}$ radiation profiles for $\mathrm{P}$ - and $\mathrm{S}$ polarization were measured and fitted (Supporting Information 1.3). The profiles from $\mathrm{LH}$ and $\mathrm{RH}$ substrates are distinctly different and the S-polarized profiles display the largest differences. The S-polarized profiles for when the front face is immersed in buffer and the fits along with the associated fitting parameters are shown in Figure 6 and Table 1 for $\mathrm{LH}$ and $\mathrm{RH}$ substrates (unstructured $\mathrm{Au}$ film is also shown for comparison). Within the precision of the measurements only the $\alpha, \beta$, and $\delta$ parameters $\left(E_{1}, E_{2}\right.$, and $M_{1}$ contribution) differ between the $\mathrm{LH}$ and $\mathrm{RH}$ substrates. There is no such chiral sensitivity observed from $\mathrm{SH}$ measurement when exciting from the front face, where the fundamental beam is off resonance, Figure $2 \mathrm{C}, \mathrm{D}$. Given that the dipolar contribution originates only from the surface, the asymmetry in the $\alpha$ parameter solely reflects the differences in the chirality of the back face. Although quadrupolar, magnetic dipolar and higher order terms can also contribute to nonlinear optical activity in chiral plasmonic metamaterials, these contributions can also have a (achiral) bulk origin too, which may vary from $\mathrm{LH}$ and $\mathrm{RH}$ substrates due to minor fabrication differences. ${ }^{34}$ Consequently, asymmetries in $\beta$ and $\delta$ parameters may not fully reflect chirality.

Within the precision of the measurement, the back face far field OA-SHG profiles are insensitive to the (achiral) refractive index environment of the front face within the range $n=1-$ 1.48 (see Supporting Information 1.3). The insensitivity of OA- 
SHG to an achiral dielectric is consistent with the results of EM modeling (Supporting Information 1.3). Specifically, the intensity, spatial, and chiral properties of the evanescent fields in the vicinity of the back face are not significantly changed by altering the refractive index of the volume covering the front face. However, it has been previously demonstrated that the (chiral) fields of a chiral TPS are altered by changes in the level of hybridization between electric and magnetic modes of the solid and inverse structures. ${ }^{13}$ To summarize, while the (chiral) fields, and hence SHG measurements, are insensitive to the presence of a (chiral) dielectric in the vicinity of the front face, they can detect the indirect effects that the (chiral) dielectric has on the level of hybridization. Thus, OA-SHG measurements, and specifically asymmetries in the intrinsically surface specific $\alpha$-parameter, are sensitive to change in hybridization/ chirality induced by biomolecule adsorption at the front face.

OA-SHG measurements were performed with a wider range of chiral biomolecules than were used for the linear measurements. Six types of proteins were studied, two with predominately $\alpha$-helical structure (native BSA and Hemoglobin), three with high $\beta$-sheet (Con A, IgG, and $\beta$-lactoglobulin) content, and a disordered structure (denatured-BSA), see Supporting Information 1.4. Apart from proteins, model $\alpha$ helical structures produced from $\mathrm{L}$ - and $\mathrm{D}$-polylysine in basic $(\mathrm{pH}=11.5)$ solutions were also investigated. ${ }^{35}$ The polylysine helices are ideal model systems that are structurally simpler than the high $\alpha$-helical content proteins and also enable both enantiomers to be studied (Supporting Information 1.4). Because all proteins are composed of L-amino acids, Dpolylysine produces the non-natural $\alpha$-helix enantiomer. For brevity, the OA-SHG experimental far-field radiation profiles with associated fits for $\mathrm{RH}$ and $\mathrm{LH}$ gammadions, for three proteins representative of the structural motifs, adsorbed on the front face, are found in Figure 7. Plots for all the proteins and fitting parameters available in Supporting Information 1.4. Although three fitting parameters $(\alpha, \beta, \delta)$ display sensitivity to nanostructure chirality, only the $\alpha$ (dipolar contribution) parameter is measurably changed upon the adsorption of the chiral biomolecules. The change in only the dipolar contribution to the OA-SHG response indicates that the adsorption of the biomolecules on the front face alters the chiral properties of the back face surface.

Changes in the $\alpha$ parameter induced by biomolecule adsorption are quantified by calculating an asymmetry parameter

$$
\zeta=\left(\frac{\left(\alpha_{\text {Biomolecule }}-\alpha_{\text {Buffer }}\right)}{\left(\alpha_{\text {Buffer }}\right)}\right)
$$

for both gammadion enantiomers, Table 2. The L- and Dpolylysine display the expected enantiomer dependence of a chiral phenomenon with equal and opposite effects being observed. The adsorption of L-polylysine (natural $\alpha$-helix) only causes a significant change to the far field $\mathrm{SH}$ profiles of the $\mathrm{RH}$ substrate with a $\zeta=0.26 \pm 0.03$, while D-polylysine (nonnatural $\alpha$-helix) only changes the LH profile significantly with a $\zeta=0.25 \pm 0.03$.

The protein data in Table 2 clearly demonstrate that the $\zeta$ parameter displays sensitivity to the secondary structure content of proteins, which goes beyond that observed in superchiral polarimetry. As would be expected, the $\zeta$ parameters for the high $\alpha$-helical content proteins display similar behavior to the L-polylysine $\alpha$-helix. Importantly, the $\zeta$ -
Table 2. Asymmetry Values Calculated from the Fits to the SHG Experimental Results for the Protein Solutions

\begin{tabular}{|c|c|c|c|}
\hline & & \multicolumn{2}{|c|}{$\zeta$} \\
\hline & & Left & Right \\
\hline \multirow{4}{*}{$\alpha$-helix } & Poly L-Lysine & $0.00 \pm 0.03$ & $0.25 \pm 0.03$ \\
\hline & Poly D-Lysine & $0.26 \pm 0.03$ & $0.04 \pm 0.03$ \\
\hline & BSA & $0.02 \pm 0.03$ & $0.22 \pm 0.03$ \\
\hline & Haemoglobin & $0.00 \pm 0.03$ & $0.14 \pm 0.03$ \\
\hline \multirow{3}{*}{$\beta$-sheet \{} & Concanavalin A & $-0.06 \pm 0.03$ & $-0.20 \pm 0.03$ \\
\hline & $\beta$ - Lactoglobulin & $0.06 \pm 0.03$ & $-0.18 \pm 0.03$ \\
\hline & $\lg G$ & $0.00 \pm 0.03$ & $-0.20 \pm 0.03$ \\
\hline isordered & BSA Denatured & $-0.04 \pm 0.03$ & $-0.04 \pm 0.03$ \\
\hline
\end{tabular}

parameter provides a distinct fingerprint of the three secondary structural motifs ( $\alpha$-helix, $\beta$-sheet, and disordered structure); $\beta$ sheet displays a negative $\zeta$-value for $\mathrm{RH}$ and no change for $\mathrm{LH}$, whereas for disordered BSA there is no asymmetry for the $\zeta$ values for $\mathrm{LH}$ and $\mathrm{RH}$ substrates.

The change in shape in the Fano resonance in the front face reflectance spectra and the commensurate change in the chiral properties of the surface of the back face, detected by OA-SHG, support the hypothesis that the adsorption of biomolecules mediates the hybridization of the chiral electric and magnetic modes of the solid-inverse structure. The novel effect reported is a chiral phenomenon that is dependent on both the handedness of the primary structure (i.e., chirality of amino acid units), as well as the secondary structure, of the biomolecules and the near fields. The novel phenomenon observed is distinctly different from previous reported superchiral polarimetry. It is observed when no superchiral polarimetry asymmetry (i.e., an asymmetry in the effective refractive indices in the presence of a chiral field) is observed under identical conditions. Crucially, OA-SHG data indicates that the phenomenon is observed for subchiral fields $(C<1)$. The new phenomenon is also a more incisive probe of biomacromolecular structure than superchiral polarimetry providing distinct fingerprints in OA-SHG of the three secondary structure motifs.

The mechanism for the observed phenomenon is clearly different to that of superchiral polarimetry. The large measurable asymmetries in superchiral polarimetry arise because its chiral sensitivity derives from the quadrupolar response of a medium enabling it to discriminate between structurally anisotropic and isotropic biomolecules. ${ }^{10}$ The different characteristics of the current effect, in particular the sensitivity of approximately structurally isotropic proteins (BSA and Hemoglobin) and greater structural incisiveness, indicates that the observed chiral dependency is not quadrupolar in origin. Thus, the ability of a biomolecule to mediate the hybridization of electric and magnetic modes must be associated with the $E_{1} M_{1}$ response. It is the $E_{1} M_{1}$ cross term that is responsible for chiral sensitivity of traditional chiroptical techniques such as UV/visible and vibrational circular dichroism spectroscopies in isotropic samples. ${ }^{12}$ This greater structural incisiveness is consistent with the observations. Intuitively, one would predict that the $\mathrm{E}_{1} \mathrm{M}_{1}$ cross term would mediate the hybridization of the electric and magnetic modes of the solid and inverse structures because it is this term that describes the combined interaction of electric and magnetic fields with matter. 
In summary, we demonstrate a new plasmonic phenomena, the mediation of the hybridization of chiral electric and magnetic modes in a solid-inverse nanostructure by chiral molecular media. The effect enables ultrasensitive detection (Supporting Information 1.5) and characterization of biomaterials without reliance on either refractive index sensitivity or the creation of near fields with enhanced chiral asymmetries. The dependency of the phenomenon on the $E_{1} M_{1}$ response of a material makes it an incisive probe of biomacromolecular structure. This offers an alternative paradigm to polarimetry with either light or superchiral evanescent fields for the ultrasensitive characterization of biomaterials. The fact that weakly chiral fields can be used allows greater flexibility in metamaterial design, lifting constraints such as the need to engineer structures that produce superchiral fields. In a much broader context, our work reveals a new approach for engineering the optical properties of the metamaterials. It demonstrates that molecular media can affect the hybridization of modes in a structure, which to date would otherwise have been achieved through controlling nanostructure geometry. Given the potential for controllable molecular functionality our study provides a glimpse of a new chemical strategy for the metamaterial design toolbox.

\section{ASSOCIATED CONTENT}

\section{S Supporting Information}

The Supporting Information is available free of charge on the ACS Publications website at DOI: 10.1021/acs.nanolett.6b02549.

Materials and methods, symmetry analysis, SHG experimental setup, TPS characterization and properties, protein experiments, detected protein mass (PDF)

\section{AUTHOR INFORMATION}

\section{Corresponding Authors}

*E-mail: Affar.Karimullah@glasgow.ac.uk.

*E-mail: Malcolm.Kadodwala@glasgow.ac.uk.

\section{Notes}

The authors declare no competing financial interest.

\section{ACKNOWLEDGMENTS}

The authors acknowledge financial support from the Engineering and Physical Sciences Research Council (EPSRC EP/ K034936/1), National Science Foundation (NSF Grant CHE1307021), JSPS Core to Core, and the technical support from the James Watt Nanofabrication Centre (JWNC). R.T. and C.J. thank the EPSRC for the award of scholarships.

\section{REFERENCES}

(1) Lal, S.; Link, S.; Halas, N. J. Nano-Optics from Sensing to Waveguiding. Nat. Photonics 2007, 1, 641-648.

(2) Cooper, M. A. Optical Biosensors in Drug Discovery. Nat. Rev. Drug Discovery 2002, 1, 515-528.

(3) Jack, C.; Karimullah, A. S.; Tullius, R; Khorashad, L. K.; Rodier, M.; Fitzpatrick, B.; Barron, L. D.; Gadegaard, N.; Lapthorn, A. J.; Rotello, V. M.; et al. Spatial Control of Chemical Processes on Nanostructures through Nano-Localized Water Heating. Nat. Commun. 2016, 7, 10946.

(4) Circular Dichroism and the Conformational Analysis of Biomolecules; Fasman, G. D., Ed.; Springer: Boston, MA, 1996.

(5) Tang, Y.; Cohen, A. E. Enhanced Enantioselectivity in Excitation of Chiral Molecules by Superchiral Light. Science 2011, 332, 333-336.
(6) Zhang, H.; Govorov, A. O. Giant Circular Dichroism of a Molecule in a Region of Strong Plasmon Resonances between Two Neighboring Gold Nanocrystals. Phys. Rev. B: Condens. Matter Mater. Phys. 2013, 87, 075410.

(7) Govorov, A. O. Plasmon-Induced Circular Dichroism of a Chiral Molecule in the Vicinity of Metal Nanocrystals. Application to Various Geometries. J. Phys. Chem. C 2011, 115, 7914-7923.

(8) Govorov, A. O.; Fan, Z.; Hernandez, P.; Slocik, J. M.; Naik, R. R. Theory of Circular Dichroism of Nanomaterials Comprising Chiral Molecules and Nanocrystals: Plasmon Enhancement, Dipole Interactions, and Dielectric Effects. Nano Lett. 2010, 10, 1374-1382.

(9) Maoz, B. M.; Chaikin, Y.; Tesler, A. B.; Bar Elli, O.; Fan, Z.; Govorov, A. O.; Markovich, G. Amplification of Chiroptical Activity of Chiral Biomolecules by Surface Plasmons. Nano Lett. 2013, 13, 12031209.

(10) Hendry, E.; Carpy, T.; Johnston, J.; Popland, M.; Mikhaylovskiy, R. V.; Lapthorn, A. J.; Kelly, S. M.; Barron, L. D.; Gadegaard, N.; Kadodwala, M. Ultrasensitive Detection and Characterization of Biomolecules Using Superchiral Fields. Nat. Nanotechnol. 2010, 5, 783-787.

(11) Tullius, R.; Karimullah, A. S.; Rodier, M.; Fitzpatrick, B.; Gadegaard, N.; Barron, L. D.; Rotello, V. M.; Cooke, G.; Lapthorn, A.; Kadodwala, M. Superchiral" Spectroscopy: Detection of Protein Higher Order Hierarchical Structure with Chiral Plasmonic Nanostructures. J. Am. Chem. Soc. 2015, 137, 8380-8383.

(12) Barron, L. D. Molecular Light Scattering and Optical Activity; 2nd ed.; Cambridge University Press: New York, 2009.

(13) Karimullah, A. S.; Jack, C.; Tullius, R.; Rotello, V. M.; Cooke, G.; Gadegaard, N.; Barron, L. D.; Kadodwala, M. Disposable Plasmonics: Plastic Templated Plasmonic Metamaterials with Tunable Chirality. Adv. Mater. 2015, 27, 5610-5616.

(14) Hentschel, M.; Weiss, T.; Bagheri, S.; Giessen, H. Babinet to the Half: Coupling of Solid and Inverse Plasmonic Structures. Nano Lett. 2013, 13, 4428-4433.

(15) Prodan, E.; Radloff, C.; Halas, N. J.; Nordlander, P. A Hybridization Model for the Plasmon Response of Complex Nanostructures. Science 2003, 302, 419-422.

(16) Davis, T. J.; Gómez, D. E.; Vernon, K. C. Simple Model for the Hybridization of Surface Plasmon Resonances in Metallic Nanoparticles. Nano Lett. 2010, 10, 2618-2625.

(17) Wang, H.; Brandl, D. W.; Nordlander, P.; Halas, N. J. Plasmonic Nanostructures: Artificial Molecules. Acc. Chem. Res. 2007, 40, 53-62.

(18) Hao, F.; Sonnefraud, Y.; Dorpe, P.; Maier, S. A.; Halas, N. J.; Nordlander, P. Symmetry Breaking in Plasmonic Nanocavities: Subradiant LSPR Sensing and a Tunable Fano Resonance. Nano Lett. 2008, 8, 3983-3988.

(19) Liu, N.; Langguth, L.; Weiss, T.; Kästel, J.; Fleischhauer, M.; Pfau, T.; Giessen, H. Plasmonic Analogue of Electromagnetically Induced Transparency at the Drude Damping Limit. Nat. Mater. 2009, $8,758-762$

(20) Zhang, S.; Genov, D. A.; Wang, Y.; Liu, M.; Zhang, X. PlasmonInduced Transparency in Metamaterials. Phys. Rev. Lett. 2008, 101, 047401.

(21) Miroshnichenko, A. E.; Flach, S.; Kivshar, Y. S. Fano Resonances in Nanoscale Structures. Rev. Mod. Phys. 2010, 82, $2257-2298$

(22) Luk'yanchuk, B.; Zheludev, N. I.; Maier, S. A.; Halas, N. J.; Nordlander, P.; Giessen, H.; Chong, C. T. The Fano Resonance in Plasmonic Nanostructures and Metamaterials. Nat. Mater. 2010, 9, $707-715$

(23) Tang, Y.; Cohen, A. E. Optical Chirality and Its Interaction with Matter. Phys. Rev. Lett. 2010, 104, 163901.

(24) Davis, T. J.; Hendry, E. Superchiral Electromagnetic Fields Created by Surface Plasmons in Nonchiral Metallic Nanostructures. Phys. Rev. B: Condens. Matter Mater. Phys. 2013, 87, 085405.

(25) Hendry, E.; Mikhaylovskiy, R. V.; Barron, L. D.; Kadodwala, M.; Davis, T. J. Chiral Electromagnetic Fields Generated by Arrays of Nanoslits. Nano Lett. 2012, 12, 3640-3644. 
(26) Butet, J.; Brevet, P.-F.; Martin, O. J. F. Optical Second Harmonic Generation in Plasmonic Nanostructures: From Fundamental Principles to Advanced Applications. ACS Nano 2015, 9, 10545-10562.

(27) Butet, J.; Martin, O. J. F. Fano Resonances in the Nonlinear Optical Response of Coupled Plasmonic Nanostructures. Opt. Express 2014, 22, 29693.

(28) Kauranen, M.; Zayats, A. V. Nonlinear Plasmonics. Nat. Photonics 2012, 6, 737-748.

(29) Valev, V. K.; Baumberg, J. J.; De Clercq, B.; Braz, N.; Zheng, X.; Osley, E. J.; Vandendriessche, S.; Hojeij, M.; Blejean, C.; Mertens, J.; et al. Nonlinear Superchiral Meta-Surfaces: Tuning Chirality and Disentangling Non-Reciprocity at the Nanoscale. Adv. Mater. 2014, 26, 4074-4081.

(30) Capretti, A.; Walsh, G. F.; Minissale, S.; Trevino, J.; Forestiere, C.; Miano, G.; Dal Negro, L. Multipolar Second Harmonic Generation from Planar Arrays of Au Nanoparticles. Opt. Express 2012, 20, $15797-15806$

(31) Abdulrahman, N.; Syme, C. D.; Jack, C.; Karimullah, A.; Barron, L. D.; Gadegaard, N.; Kadodwala, M. The Origin of off-Resonance Non-Linear Optical Activity of a Gold Chiral Nanomaterial. Nanoscale 2013, 5, 12651-12657.

(32) Dadap, J. I.; Shan, J.; Eisenthal, K. B.; Heinz, T. F. SecondHarmonic Rayleigh Scattering from a Sphere of Centrosymmetric Material. Phys. Rev. Lett. 1999, 83, 4045-4048.

(33) Russier-Antoine, I.; Benichou, E.; Bachelier, G.; Jonin, C.; Brevet, P. F. Multipolar Contributions of the Second Harmonic Generation from Silver and Gold Nanoparticles. J. Phys. Chem. C 2007, 111, 9044-9048.

(34) Zdanowicz, M.; Kujala, S.; Husu, H.; Kauranen, M. Effective Medium Multipolar Tensor Analysis of Second-Harmonic Generation from Metal Nanoparticles. New J. Phys. 2011, 13, 023025.

(35) Chiou, J.-S.; Tatara, T.; Sawamura, S.; Kaminoh, Y.; Kamaya, H.; Shibata, A.; Ueda, I. The $\alpha$-Helix to $\beta$-Sheet Transition in Poly(1Lysine): Effects of Anesthetics and High Pressure. Biochim. Biophys. Acta, Protein Struct. Mol. Enzymol. 1992, 1119, 211-217. 